\title{
Local extrema in random permutations and the structure of longest alternating subsequences
}

\author{
Dan Romik $\|^{\prime}$ \\ ${ }^{1}$ Department of Mathematics, University of California, Davis, CA, USA
}

\begin{abstract}
Let $\mathbf{a s}_{n}$ denote the length of a longest alternating subsequence in a uniformly random permutation of order $n$. Stanley studied the distribution of $\mathbf{a s}_{n}$ using algebraic methods, and showed in particular that $\mathbb{E}\left(\mathbf{a s}_{n}\right)=(4 n+1) / 6$ and $\operatorname{Var}\left(\mathbf{a s}_{n}\right)=(32 n-13) / 180$. From Stanley's result it can be shown that after rescaling, $\mathbf{a s}_{n}$ converges in the limit to the Gaussian distribution. In this extended abstract we present a new approach to the study of $\mathbf{a s}_{n}$ by relating it to the sequence of local extrema of a random permutation, which is shown to form a "canonical" longest alternating subsequence. Using this connection we reprove the abovementioned results in a more probabilistic and transparent way. We also study the distribution of the values of the local minima and maxima, and prove that in the limit the joint distribution of successive minimum-maximum pairs converges to the two-dimensional distribution whose density function is given by $f(s, t)=3(1-s) t e^{t-s}$.

Résumé. Pour une permutation aléatoire d'ordre $n$, on désigne par $\mathbf{a s}_{n}$ la longueur maximale d'une de ses sous-suites alternantes. Stanley a étudié la distribution de $\mathbf{a s}_{n}$ en utilisant des méthodes algébriques, et il a démontré en particulier que $\mathbb{E}\left(\mathbf{a s}_{n}\right)=(4 n+1) / 6$ et $\operatorname{Var}\left(\mathbf{a s}_{n}\right)=(32 n-13) / 180$. A partir du résultat de Stanley on peut montrer qu'après changement d'échelle, $\mathbf{a s}_{n}$ converge vers la distribution normale. Nous présentons ici une approche nouvelle pour l'étude de $\mathbf{a s}_{n}$, en la reliant à la suite des extrema locaux d'une permutation aléatoire, dont nous montrons qu'elle constitue une sous-suite alternante maximale "canonique". En utilisant cette relation, nous prouvons à nouveau les résultats mentionnés ci-dessus d'une façon plus probabiliste et transparente. En plus, nous prouvons un résultat asymptotique sur la distribution limite des paires formées d'un minimum et d'un maximum locaux consécutifs.
\end{abstract}

Keywords: longest alternating subsequences, permutation statistics, random permutation

\section{Introduction}

Let $x_{1}, \ldots, x_{n}$ be a sequence of distinct real numbers. A subsequence $x_{i_{1}}, \ldots, x_{i_{k}}$, where $1 \leq i_{1}<$ $\ldots<i_{k} \leq n$, is called an alternating subsequence if it satisfies

$$
x_{i_{1}}>x_{i_{2}}<x_{i_{3}}>\ldots x_{k}
$$

\footnotetext{
${ }^{\dagger}$ Supported by the National Science Foundation under grant DMS-0955584.

1365-8050 @ 2011 Discrete Mathematics and Theoretical Computer Science (DMTCS), Nancy, France
} 
Let $\operatorname{as}_{\max }\left(x_{1}, \ldots, x_{n}\right)$ be the maximal length of an alternating subsequence of $x_{1}, \ldots, x_{n}$. Note that there may be more than one alternating subsequence of this maximal length. For example, the sequence $4,2,1,3$ has three longest alternating subsequences, namely $(4,1,3),(4,2,3)$ and $(2,1,3)$.

In this extended abstract we are concerned with the random variable

$$
\mathbf{a s}_{n}=\operatorname{as}_{\max }(\sigma(1), \sigma(2), \ldots, \sigma(n)),
$$

where $\sigma$ is a uniformly random permutation in the symmetric group $S_{n}$. Equivalently, for our purposes it will be convenient to realize $\mathbf{a s}_{n}$ as

$$
\operatorname{as}_{n}=\operatorname{as}_{\max }\left(X_{1}, X_{2}, \ldots, X_{n}\right),
$$

where $X_{1}, X_{2}, \ldots, X_{n}$ is a sequence of independent and identically distributed random variables with the uniform distribution $U[0,1]$. The fact that this realization gives a random variable with the same distribution is a consequence of the well-known fact that the order structure of $\left(X_{1}, \ldots, X_{n}\right)$ is a uniformly random element of $S_{n}$.

Stanley (2008) studied $\mathbf{a s}_{n}$, and proved, among other results, the following exact formulas for the expectation and variance of $\mathbf{a s}_{n}$ :

$$
\begin{aligned}
\mathbb{E}\left(\mathbf{a s}_{n}\right) & =\frac{2}{3} n+\frac{1}{6}, & & (n \geq 2), \\
\operatorname{Var}\left(\mathbf{a s}_{n}\right) & =\frac{8}{45} n-\frac{13}{180}, & & (n \geq 4) .
\end{aligned}
$$

In particular, for large values of $n, \mathbf{a s}_{n}$ takes values which are with high probability concentrated around its mean value of approximately $2 n / 3$, with a standard deviation of order $\sqrt{n}$. Stanley also noted that the distribution of $\mathbf{a s}_{n}$ converges in the limit as $n \rightarrow \infty$ to the Gaussian distribution. This was proved by Widom (2006) using a generating function identity derived by Stanley. Stanley also pointed out (see also Stanley (2010)) that the limiting Gaussian distribution follows using the same generating function identity from general results of Pemantle and Wilson (2002), and sketches a different proof based on unpublished results of Wilf (1998), which also rely on properties of specific generating functions related to $\mathbf{a s}_{n}$.

In this extended abstract, we present a new approach to the study of the distribution of $\mathbf{a s}_{n}$. The main new idea is that one can construct a specific longest alternating subsequence of a given sequence $x_{1}, \ldots, x_{n}$ in a simple way using the "local extrema" of the sequence. In the probabilistic setting, the length $\mathbf{a s}_{n}$ is then equal (modulo some boundary corrections) to the number of local extrema of the sequence of random variables $X_{1}, \ldots, X_{n}$. This number can be represented explicitly as a sum of Bernoulli random variables with simple correlations, which leads immediately to a new and more transparent derivation of the relations (2), (3) and the limiting Gaussian distribution. Furthermore, with this approach it is natural to try to understand the structure of this distinguished longest alternating subsequence formed from the local extrema; we will derive explicit formulas for the limiting densities of the local minima and maxima, and for the limiting two-dimensional density of "minimum-maximum pairs", which consist of a local minimum and the local maximum that follows it.

\section{Local extrema and the canonical alternating subsequence}

Let us start with the combinatorial description of the sequence of local extrema and its relation to longest alternating subsequences. Let $x_{1}, \ldots, x_{n}$ be a sequence of distinct numbers. For $2 \leq k \leq n-1$, 
we say that $x_{k}$ is a local minimum if $x_{k-1}>x_{k}<x_{k+1}$. We say that $x_{k}$ is a local maximum if $x_{k-1}<x_{k}>x_{k+1}$, and we say that it is a local extremum if it is a local minimum or maximum. Define the canonical alternating subsequence to be the sequence of local extrema of $x_{1}, \ldots, x_{n}$, together with the last element $x_{n}$, and together with the first element $x_{1}$ if it satisfies $x_{1}>x_{2}$.

Lemma 1 The canonical alternating subsequence is in fact an alternating subsequence, and its length is $\operatorname{as}_{\max }\left(x_{1}, \ldots, x_{n}\right)$.

Proof: Because local minima and maxima appear in alternation, the sequence of local extrema meets the definition of an alternating subsequence, except possibly the requirement that the subsequence starts with a descent rather than an ascent. To make sure this requirement is also met, we add $x_{1}$ to the subsequence if the first local extremum is a minimum, which happens exactly if $x_{1}>x_{2}$. It is also easy to check that adding $x_{n}$ does not damage the alternating property in any case, so the canonical subsequence is alternating.

Denote the canonical alternating subsequence by $x_{j_{1}}, \ldots, x_{j_{m}}$, and denote $A=\operatorname{as}_{\max }\left(x_{1}, \ldots, x_{n}\right)$. By definition we have that $m \leq A$. Conversely, to show that $m \geq A$, observe that the sequence of elements between successive local extrema is necessarily monotone. Therefore any alternating subsequence can contain at most one index from each of $\left[1, j_{1}\right),\left[j_{1}, j_{2}\right), \ldots,\left[j_{m-1}, j_{m}\right)$ and $\left[j_{m}, n\right]=\{n\}$ and therefore has length $\leq m$.

\section{A new probabilistic representation of $\mathbf{a s}_{n}$}

As a corollary to the last result, we get the following convenient representation for the random variable $\mathbf{a s}_{n}$ as defined in (1).

Corollary 2 Define events $A_{1}, \ldots, A_{n-1}$ depending on the random variables $X_{1}, \ldots, X_{n}$ by

$$
\begin{aligned}
& A_{1}=\left\{X_{1}>X_{2}\right\} \\
& A_{k}=\left\{X_{k-1}<X_{k}>X_{k+1}\right\} \cup\left\{X_{k-1}>X_{k}<X_{k+1}\right\}, \quad(2 \leq k \leq n-1) .
\end{aligned}
$$

Let $I_{A}$ denote the indicator random variable of an event $A$. Then we have

$$
\mathbf{a s}_{n}=1+\sum_{k=1}^{n-1} I_{A_{k}} .
$$

Proof: For $2 \leq k \leq n-1, A_{k}$ represents the event that $X_{k}$ is a local extremum in the sequence $X_{1}, \ldots, X_{n}$. So, the right-hand side of (4) exactly counts the number of terms in the canonical alternating subsequence.

\section{Computation of the mean and variance}

Next, we compute the expectations of the indicator random variables $I_{A_{k}}, 1 \leq k \leq n-1$, and their correlations. 
Lemma 3 The means of the indicator random variables $I_{A_{k}}, 1 \leq k \leq n-1$, are given by

$$
\mathbb{E}\left(I_{A_{k}}\right)=\mathbb{P}\left(A_{k}\right)= \begin{cases}1 / 2 & k=1 \\ 2 / 3 & 2 \leq k \leq n-1\end{cases}
$$

The covariances $\operatorname{Cov}\left(I_{A_{j}}, I_{A_{k}}\right)=\mathbb{E}\left(I_{A_{j}} I_{A_{k}}\right)-\mathbb{E}\left(I_{A_{j}}\right) \mathbb{E}\left(I_{A_{k}}\right), \quad 1 \leq j, k \leq n-1$, are given in the following covariance matrix:

$$
\left(\operatorname{Cov}\left(I_{A_{j}}, I_{A_{k}}\right)\right)_{j, k=1}^{n-1}=\left(\begin{array}{cccccccccc}
\frac{1}{4} & 0 & 0 & 0 & 0 & 0 & 0 & 0 & \ldots & 0 \\
0 & \frac{2}{9} & \frac{-1}{36} & \frac{1}{180} & 0 & 0 & 0 & 0 & \ldots & 0 \\
0 & \frac{-1}{36} & \frac{2}{9} & \frac{-1}{36} & \frac{1}{180} & 0 & 0 & 0 & \ldots & 0 \\
0 & \frac{1}{180} & \frac{-1}{36} & \frac{2}{9} & \frac{-1}{36} & \frac{1}{180} & 0 & 0 & \ldots & 0 \\
0 & 0 & \frac{1}{180} & \frac{-1}{36} & \frac{2}{9} & \frac{-1}{36} & \frac{1}{180} & 0 & \cdots & 0 \\
0 & 0 & 0 & \frac{1}{180} & \frac{-1}{36} & \frac{2}{9} & \frac{-1}{36} & \frac{1}{180} & \ldots & 0 \\
\vdots & \vdots & & \ddots & \ddots & \ddots & \ddots & \ddots & & \vdots \\
0 & 0 & \ldots & & 0 & \frac{1}{180} & \frac{-1}{36} & \frac{2}{9} & \frac{-1}{36} & \frac{1}{180} \\
0 & 0 & \ldots & & & 0 & \frac{1}{180} & \frac{-1}{36} & \frac{2}{9} & \frac{-1}{36} \\
0 & 0 & \ldots & & & & 0 & \frac{1}{180} & \frac{-1}{36} & \frac{2}{9}
\end{array}\right) .
$$

Proof: The computation of the means is trivial and is omitted. For the covariances, note that the events $A_{j}$ and $A_{k}$ are independent (and therefore the corresponding covariance is 0 ) if $|j-k|>2$, since each $A_{j}$ is a function of $X_{j-1}, X_{j}$ and $X_{j+1}$ only; this leaves the diagonal strip $|j-k| \leq 2$ containing potentially non-zero terms, which are computed as follows. By symmetry assume that $j \leq k$. On the main diagonal $j=k$, it is easy to compute the variances

$$
\operatorname{Cov}\left(I_{A_{k}}, I_{A_{k}}\right)=\operatorname{Var}\left(I_{A_{k}}\right)=\mathbb{P}\left(A_{k}\right)\left(1-\mathbb{P}\left(A_{k}\right)\right)= \begin{cases}1 / 4 & k=1 \\ 2 / 9 & 2 \leq k \leq n-1\end{cases}
$$

Next, if $j=1$, we have for $k=2$ that

$$
\operatorname{Cov}\left(I_{A_{1}}, I_{A_{2}}\right)=\mathbb{P}\left(A_{1} \cap A_{2}\right)-\mathbb{P}\left(A_{1}\right) \mathbb{P}\left(A_{2}\right)=\mathbb{P}\left(X_{1}>X_{2}<X_{3}\right)-\frac{1}{2} \cdot \frac{2}{3}=\frac{1}{3}-\frac{1}{3}=0,
$$

and similarly for $k=3$,

$$
\begin{aligned}
\operatorname{Cov}\left(I_{A_{1}}, I_{A_{3}}\right) & =\mathbb{P}\left(A_{1} \cap A_{3}\right)-\frac{1}{2} \cdot \frac{2}{3}=\mathbb{P}\left(X_{1}>X_{2}>X_{3}<X_{4}\right)+\mathbb{P}\left(X_{1}>X_{2}<X_{3}>X_{4}\right)-\frac{1}{3} \\
& =\frac{1}{8}+\frac{5}{24}-\frac{1}{3}=0
\end{aligned}
$$


(The number $5 / 24$ comes from the fact that there are 5 alternating permutations of order 4 .) Next, if $k=j+1 \geq 3$, we have

$$
\begin{aligned}
\operatorname{Cov}\left(I_{A_{j}}, I_{A_{j+1}}\right) & =\mathbb{P}\left(A_{2} \cap A_{3}\right)-\frac{2}{3} \cdot \frac{2}{3} \\
& =\mathbb{P}\left(X_{1}>X_{2}<X_{3}>X_{4}\right)+\mathbb{P}\left(X_{1}<X_{2}>X_{3}<X_{4}\right)-\frac{4}{9}=\frac{10}{24}-\frac{4}{9}=\frac{-1}{36} .
\end{aligned}
$$

Finally, for $k=j+2 \geq 4$ we have

$$
\begin{aligned}
\operatorname{Cov}\left(I_{A_{j}}, I_{A_{j+2}}\right)= & \mathbb{P}\left(A_{2} \cap A_{4}\right)-\frac{2}{3} \cdot \frac{2}{3} \\
= & \mathbb{P}\left(X_{1}<X_{2}>X_{3}<X_{4}>X_{5}\right)+\mathbb{P}\left(X_{1}>X_{2}<X_{3}>X_{4}<X_{5}\right) \\
& +\mathbb{P}\left(X_{1}<X_{2}>X_{3}>X_{4}<X_{5}\right)+\mathbb{P}\left(X_{1}>X_{2}<X_{3}<X_{4}>X_{5}\right)-\frac{4}{9} \\
= & 2 \cdot \frac{16}{120}+2 \cdot \frac{11}{120}-\frac{4}{9}=\frac{1}{180},
\end{aligned}
$$

where the fraction $16 / 120$ comes from the fact that there are 16 alternating permutations of order 5 , and the fraction $11 / 120$ comes from the fact that there are 11 permutations $\sigma$ of order 5 satisfying the order relations

$$
\sigma(1)<\sigma(2)>\sigma(3)>\sigma(4)<\sigma(5)
$$

(see also Lemma6 below for an alternative way to compute this number).

Using Lemma 3 and the representation (4), we can now easily compute the mean and variance of $\mathbf{a s}_{n}$ to recover the relations (2), (3):

$$
\begin{aligned}
\mathbb{E}\left(\mathbf{a s}_{n}\right) & =\mathbb{E}\left(1+\sum_{k=1}^{n-1} I_{A_{k}}\right)=1+\frac{1}{2}+(n-2) \frac{2}{3}=\frac{2}{3} n+\frac{1}{6}, \\
\operatorname{Var}\left(\mathbf{a s}_{n}\right) & =\operatorname{Var}\left(1+\sum_{k=1}^{n-1} I_{A_{k}}\right)=\sum_{j, k=1}^{n} \operatorname{Cov}\left(I_{A_{j}}, I_{A_{k}}\right) \\
& =\frac{1}{4}+(n-2) \frac{2}{9}+2(n-3) \frac{-1}{36}+2(n-4) \frac{1}{180}=\frac{8}{45} n-\frac{13}{180},
\end{aligned}
$$

where in the computation of the mean we assume that $n \geq 2$, and for the variance computation we assume that $n \geq 4$. Note that in principle, higher moments of $\mathbf{a s}_{n}$ can also be computed in the same way, although the computations require higher-order correlations (e.g., of the form $\mathbb{E}\left(I_{A_{j}} I_{A_{k}} I_{A_{\ell}}\right)$ ) and therefore become more tedious.

\section{The limiting Gaussian distribution}

We now show how the representation (4) can be used to deduce the limiting Gaussian distribution of $\mathbf{a s}_{n}$.

Proposition 4 For all $t \in \mathbb{R}$ we have

$$
\mathbb{P}\left(\frac{\mathbf{a s}_{n}-\mathbb{E}\left(\mathbf{a s}_{n}\right)}{\left(\operatorname{Var}\left(\mathbf{a s}_{n}\right)\right)^{1 / 2}} \leq t\right) \rightarrow \frac{1}{\sqrt{2 \pi}} \int_{-\infty}^{t} e^{-x^{2} / 2} d x \quad \text { as } n \rightarrow \infty
$$


Proof: First, note that in (4) we can ignore the constant 1 and the first summand $I_{A_{1}}$ in the sum of indicators, since their contribution is negligible compared to the scale of $\sqrt{n}$. Thus, if we consider the modified random variable $\mathbf{a s}_{n}^{\prime}=\sum_{k=2}^{n-1} I_{A_{k}}$, it will be enough to prove that $\mathbf{a s}_{n}^{\prime}$ converges after scaling to the standard Gaussian distribution $N(0,1)$. This sum is a sum of a sequence of identically distributed random variables. The sequence of random variables is not independent, but it is "3-dependent", meaning that any two contiguous blocks of random variables that are separated by a gap of length 3 or more are independent of each other. It is a well-known fact that for bounded and identically distributed random variables (and even under much weaker conditions), one can replace the condition of independence by " $m$-dependence" (where $m$ is the size of the gap needed to ensure independence of the two blocks), and this is enough to ensure that the standard central limit theorem from probability theory holds. Formally, our sequence satistfies the assumptions of Theorem 1 of Hoeffding and Robbins (1948) (whose proof is based on a simple reduction to the standard central limit theorem for independent sequences), which gives the desired conclusion on convergence to the Gaussian distribution.

We emphasize that, while the proofs of the convergence to Gaussian distribution given by Stanley (2008) and Widom (2006) also rely on fairly straightforward techniques, namely asymptotic analysis of generating functions, they provide little intuition regarding why one should expect to see a Gaussian limit. The proof above makes the situation much clearer, since it is based on the observation that $\mathbf{a s}_{n}$ is a sum of many weakly independent components.

\section{The distribution of local extrema}

Having demonstrated the relevance of the sequence of local extrema to understanding the longest alternating subsequence statistic as ${ }_{n}$, it now makes sense to try to understand aspects of the behavior of the extrema, other than just how many of them there are. In particular, it is natural to look at the distribution of the extrema values - i.e., how small or large can we expect them to be? Of course, the extrema consist of local minima and local maxima appearing in alternation, so it makes sense to answer this question for the minima and maxima separately. The precise result is as follows.

Theorem 5 Let $N_{\min }$ be the (random) number of local minima in the sequence of random variables $X_{1}, \ldots, X_{n}$, and let $m_{1}, m_{2}, \ldots, m_{N_{\min }}$ denote the values of the local minima in the sequence, i.e., $m_{j}=X_{k_{j}}$ where $k_{j}$ is the position where the $j$-th local minimum appears. For all $0 \leq a<b \leq 1$ we have the convergence in probability

$$
\frac{1}{N_{\min }} \#\left\{1 \leq j \leq N_{\min }: m_{j} \in[a, b]\right\} \underset{n \rightarrow \infty}{\stackrel{P}{\longrightarrow}} \int_{a}^{b} 3(1-t)^{2} d t=(1-a)^{3}-(1-b)^{3} .
$$

In other words, the local minima in the limit are distributed in $[0,1]$ according to the limiting probability density function $3(1-t)^{2}$. Similarly (and symmetrically), if we denote by $N_{\max }$ the number of local maxima, and denote the values of the local maxima by $M_{1}, \ldots, M_{N_{\max }}$, then for all $0 \leq a<b \leq 1$ we have the convergence in probability

$$
\frac{1}{N_{\max }} \#\left\{1 \leq j \leq N_{\max }: M_{j} \in[a, b]\right\} \underset{n \rightarrow \infty}{\stackrel{P}{\longrightarrow}} \int_{a}^{b} 3 t^{2} d t=b^{3}-a^{3} .
$$

That is, the local maxima are in the limit distributed according to the density $3 t^{2}$. 
Proof: First, note that $N_{\min }$ and $N_{\max }$ differ by at most 1 , and their sum is equal to $\mathbf{a s}_{n}-1$ or $\mathbf{a s}_{n}-2$ (depending on whether the term $I_{A_{1}}$ in (4) is 0 or 1), so, by the results on the mean and variance of $\mathbf{a s}_{n}$, we see that both $N_{\min }$ and $N_{\max }$ are equal to $n / 3+o(n)$ with asymptotically high probability as $n \rightarrow \infty$. Second, note that it is enough to prove (5) (which by symmetry implies (6) in the case $b=1$. Next, observe that we can write the number of local minima falling in the interval $[a, 1]$ as a sum of indicator random variables, namely

$$
\#\left\{1 \leq j \leq N_{\min }: m_{j} \in[a, 1]\right\}=\sum_{k=2}^{n-1} 1_{\left.\left\{X_{k-1}>X_{k}<X_{k+1}, X_{k} \geq a\right)\right\}}
$$

It is trivial to compute the mean of each of these (identically distributed) indicators. It is equal to

$$
\mathbb{E}\left(1_{\left.\left\{X_{k-1}>X_{k}<X_{k+1}, X_{k} \geq a\right)\right\}}\right)=\mathbb{P}\left(X_{1}, X_{2}, X_{3} \geq a, X_{2}=\min \left(X_{1}, X_{2}, X_{3}\right)\right)=\frac{1}{3}(1-a)^{3} .
$$

It is also easy to see that the variance of the sum of the indicators is $O(n)$, since, as before, each of the indicators is correlated with only the two adjacent ones on each side. Therefore we get (for example using Chebyshev's inequality) that as $n \rightarrow \infty$ the left-hand side of $(7)$ is with high probability equal to $\frac{1}{3}(n-2)(1-a)^{3}+o(n)$ (in fact, the error is of order $O\left(n^{1 / 2}\right)$, and one can use the standard results from probability theory mentioned above to get a limiting Gaussian convergence for this random variable as well). Combining this with the previous observation about the asymptotic behavior of $N_{\min }$ gives (5).

\section{The joint distribution of a minimum-maximum pair}

Our final result concerns a formula for the limiting joint distribution of a local minimum and the local maximum that follows it. We start with a lemma.

Lemma 6 Let $d \geq 4$, and let $A$ be an open subset of $\{(s, t): 0 \leq s \leq t \leq 1\}$. We have

$$
\begin{aligned}
\mathbb{P}\left(X_{1}>X_{2}<X_{3}<\ldots<X_{d-1}>\right. & \left.X_{d},\left(X_{2}, X_{d-1}\right) \in A\right) \\
& =\frac{1}{(d-4) !} \iint_{A}(1-s) t(t-s)^{d-4} d s d t .
\end{aligned}
$$

Proof: The probability on the left-hand side can be expressed in an obvious way as a $d$-dimensional multiple integral of the constant function 1 on the subset of $[0,1]^{d}$ consisting of vectors $\left(x_{1}, \ldots, x_{d}\right)$ satisfying $x_{1}>x_{2}<\ldots<x_{d-1}>x_{d}$ and $\left(x_{2}, x_{d-1}\right) \in A$. Choosing $x_{2}$ and $x_{d-1}$ as the outer variables of integration, we can compute this integral as the iterated integral

$$
\begin{array}{r}
\iint_{A}\left(\int_{x_{2}}^{1} d x_{1} \int_{0}^{x_{d-1}} d x_{d} \iint \ldots \int_{\left\{x_{2} \leq x_{3} \leq \ldots \leq x_{d-2} \leq x_{d-1}\right\}} d x_{3} \ldots d x_{d-2}\right) d x_{2} d x_{d-1} \\
=\iint_{A}\left(1-x_{2}\right) x_{d-1} \frac{\left(x_{d-1}-x_{2}\right)^{d-4}}{(d-4) !} d x_{2} d x_{d-1}
\end{array}
$$

This is equal to the right-hand side of [8].

The result on the joint distribution of a minimum-maximum pair is as follows. 
Theorem 7 Let $A$ be an open subset of $\{(s, t): 0 \leq s \leq t \leq 1\}$. Denote by $N_{\text {min-max }}$ the number of minimum-maximum pairs, which are defined as pairs $(i, j)$ of positions where $i<j, X_{i}$ is a local minimum of the sequence $X_{1}, \ldots, X_{n}, X_{j}$ is a local maximum, and $X_{i}$ and $X_{j}$ are not separated by another local extremum. Denote the values of these minimum-maximum pairs $\left(X_{i}, X_{j}\right)$ (arranged in order of their appearance) by $\left(m_{1}, \mu_{1}\right),\left(m_{2}, \mu_{2}\right), \ldots,\left(m_{N_{\text {min-max }}}, \mu_{N_{\text {min-max }}}\right)$ (in the notation of Theorem 5 . $\mu_{j}=M_{j}$ or $M_{j+1}$ depending on whether the first local maximum appears after the first local minimum or before it). Then we have the convergence in probability

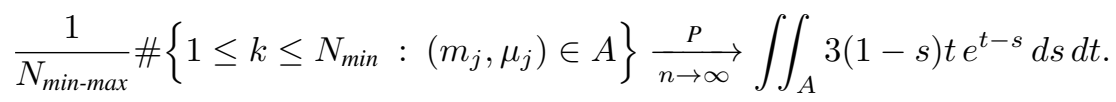

That is, the joint distribution of a local minimum-maximum pair is represented in the limit by the density function $f(s, t)=3(1-s) t e^{t-s},(0<s<t<1)$.

Proof: It is easy to see that $N_{\min -\max }$ differs from $N_{\min }$ by at most 1 , so as before, we know that it is with high probability approximately equal to $n / 3+o(n)$. Denote

$$
T_{n}=\#\left\{1 \leq k \leq N_{\text {min-max }}:\left(m_{j}, \mu_{j}\right) \in A\right\} .
$$

The key observation is that we can decompose $T_{n}$ based on the size of the gap between the position of the local minimum and the subsequent maximum. This leads to a representation

$$
T_{n}=Y_{n, 1}+Y_{n, 2}+\ldots+Y_{n, n-3},
$$

where we define random variables $Y_{n, 1}, \ldots, Y_{n, n-3}$ by

$$
\begin{aligned}
Y_{n, 1} & =\#\left\{2 \leq k \leq n-2: X_{k-1}>X_{k}<X_{k+1}>X_{k+2},\left(X_{k}, X_{k+1}\right) \in A\right\}, \\
Y_{n, 2} & =\#\left\{2 \leq k \leq n-3: X_{k-1}>X_{k}<X_{k+1}<X_{k+2}>X_{k+3},\left(X_{k}, X_{k+2}\right) \in A\right\}, \\
& \vdots \\
Y_{n, j} & =\#\left\{2 \leq k \leq n-j-1: X_{k-1}>X_{k}<X_{k+1}<\ldots<X_{k+j}>X_{k+j+1},\left(X_{k}, X_{k+j}\right) \in A\right\}, \\
& \vdots \\
Y_{n, n-3} & =\#\left\{2 \leq k \leq 2: X_{1}>X_{2}<X_{3}<\ldots<X_{n-1}>X_{n},\left(X_{2}, X_{n-1}\right) \in A\right\} .
\end{aligned}
$$

Now observe that each $Y_{n, j}$ can in turn be represented as a sum of $n-j-2$ indicator random variables of events of the form

$$
B_{n, j, k}=\left\{X_{k-1}>X_{k}<X_{k+1}<\ldots<X_{k+j}>X_{k+j+1},\left(X_{k}, X_{k+j}\right) \in A\right\} .
$$

For a fixed $j$, all of these events have the same expectation, given by the right-hand side of 8 with $d=j+3$. It follows that the expectation of $T_{n}$ is given by

$$
\mathbb{E}\left(T_{n}\right)=\sum_{j=1}^{n-3}(n-j-2) \frac{1}{(j-1) !} \iint_{A}(1-s) t(t-s)^{j-1} d s d t .
$$


Because of the fast decay of the coefficients $1 /(j-1)$ !, it is an easy exercise to sum this series asymptotically (for example by truncating it around $j \approx \log n$; see below for a related estimate), to obtain that

$$
\mathbb{E}\left(T_{n}\right)=n \iint_{A}(1-s) t e^{t-s} d s d t+O\left(n^{-10}\right) \quad \text { as } n \rightarrow \infty .
$$

So, we get that at least the mean of the variable $\frac{1}{N_{\min m a x}} T_{n}$ approaches the expression on the right-hand side of (9) in the limit. It remains to show that this random variable is concentrated around its mean. To see this, set $p_{n}=\lfloor\log n\rfloor$ (where $\lfloor x\rfloor$ denotes the integer part of $x$ ), and define

$$
T_{n}^{\prime}=\sum_{j=1}^{p_{n}} Y_{n, j}
$$

Observe that $T_{n}=T_{n}^{\prime}$ with high probability, since, by Markov's inequality,

$$
\begin{aligned}
\mathbb{P}\left(T_{n} \neq T_{n}^{\prime}\right) & =\mathbb{P}\left(\sum_{j=p_{n}+1}^{n-3} Y_{n, j}>0\right) \leq \mathbb{E}\left(\sum_{j=p_{n}+1}^{n-3} Y_{n, j}\right) \\
& \leq n \sum_{j=p_{n}+1}^{\infty} \frac{1}{(j-1) !}=O\left(n^{-10}\right) .
\end{aligned}
$$

But we know that the variance of $T_{n}^{\prime}$ is given by

$$
\operatorname{Var}\left(T_{n}^{\prime}\right)=\sum_{i, j=1}^{p_{n}} \operatorname{Cov}\left(Y_{n, i}, Y_{n, j}\right)
$$

We claim that these covariances satisfy $\operatorname{Cov}\left(Y_{n, i}, Y_{n, j}\right) \leq 10 n \log n$; if true, this implies that $\operatorname{Var}\left(T_{n}^{\prime}\right) \leq$ $10 n(\log n)^{3}$, so that $T_{n}^{\prime}$ has standard deviation of order at most $n^{1 / 2}(\log n)^{3 / 2}$ and is therefore concentrated around its mean. To see where the covariance bound comes from, consider that each $Y_{n, j}$ is represented by a sum of indicator random variables $I_{B_{n, j, k}}$, where $B_{n, j, k}$ is defined in (10) above. The number of indicators is at most $n$, and furthermore the only nonzero correlations between $I_{B_{n, j, k}}$ and $I_{B_{n, i, k^{\prime}}}$ can appear when $\left|k-k^{\prime}\right| \leq \log n$, since otherwise the indicators are functions of independent blocks of random variables from the sequence $X_{1}, \ldots, X_{n}$. This easily implies the stated covariance bound. To summarize, we have shown concentration of $T_{n}^{\prime}$ (and therefore also of $T_{n}$, by (12)) around its mean, which is also very close to the mean of $T_{n}$. Combining these facts with (11) and dividing by $n / 3$, the approximate value of $N_{\min \text {-max }}$ with high probability, gives the result.

\section{Concluding remarks}

It is interesting to contrast, as Stanley (2008) did, the results for longest alternating subsequences of random permutations with the well-developed theory of longest increasing subsequences (see Aldous and Diaconis (1999), Stanley (2007)). In all honesty, it must be admitted that the latter subject leads to a richer and more interesting theory... Still, the study of longest alternating subseqences is not without its own rewards, and provides a nice example of the interaction of algebraic-combinatorial and probabilistic 
ideas. In particular, the connection made in this extended abstract between this permutation statistic and the study of the sequence of local extrema of permutations raises interesting new questions. If one starts with a doubly-infinite sequence $\ldots, X_{-1}, X_{0}, X_{1}, X_{2}, \ldots$ of i.i.d. $U[0,1]$ random variables and considers the local extrema (so that the restriction to any finite block says something about longest alternating subsequences in that block), the sequence of local extrema can be thought of as an interesting "stationary point process" on $\mathbb{Z}$. This is analogous to the sequence of descents in random sequences, which was studied by many authors. Most recently, Borodin et al. (2010) proved that the sequence of descents is a determinantal point process. One natural question that seems worthy of further study is whether this property is shared by the sequence of local extrema.

\section{References}

D. Aldous and P. Diaconis. Longest increasing subsequences: from patience sorting to the baik-deiftjohansson. Bull. Amer. Math. Soc., 36:413-432, 1999.

A. Borodin, P. Diaconis, and J. Fulman. On adding a list of numbers (and other one-dependent determinantal processes). Bull. Amer. Math. Soc., 47:639-670, 2010.

W. Hoeffding and H. Robbins. The central limit theorem for dependent random variables. Duke Math. J., 15:773-780, 1948.

R. Pemantle and M. C. Wilson. Asymptotics of multivariate sequences: I. smooth points of the singular variety. J. Combin. Theory Ser. A, 97:129-161, 2002.

R. P. Stanley. Longest alternating subsequences of permutations. Michigan Math. J., 57:675-687, 2008.

R. P. Stanley. Increasing and decreasing subsequences and their variants. Proc. Internat. Cong. Math. Madrid 2006, pages 545-579, 2007.

R. P. Stanley. A survey of alternating permutations. Contemp. Math., to appear, 2010.

$\mathrm{H}$. Widom. On the limiting distribution for the longest alternating subsequence in a random permutation. Electron. J. Combin., 13:Article R25, 2006.

H. S. Wilf. Real zeroes of polynomials that count runs and descending runs. Unpublished preprint, 1998. 